\title{
QT prolongation in a diverse, urban population of COVID-19 patients treated with hydroxychloroquine, chloroquine, or azithromycin
}

\author{
Brian C Hsia ${ }^{1} \cdot$ Nicolas Greige $^{2}$. Jose A Quiroz ${ }^{3} \cdot$ Ahmed S Khokhar $^{3} \cdot$ Johanna Daily $^{4,5}$ • Luigi Di Biase ${ }^{1}$. \\ Kevin J Ferrick ${ }^{1} \cdot$ John D Fisher ${ }^{1} \cdot$ Andrew Krumerman $^{1}$
}

Received: 11 May 2020 / Accepted: 5 July 2020 / Published online: 11 July 2020

(C) Springer Science+Business Media, LLC, part of Springer Nature 2020

\begin{abstract}
Purpose Hydroxychloroquine, chloroquine, and azithromycin have been used for treatment of COVID-19, but may cause QT prolongation. Minority populations are disproportionately impacted by COVID-19. This study evaluates the risk of QT prolongation and subsequent outcomes after administration of these medications in largely underrepresented minority COVID-19 patients.

Methods We conducted an observational study on hospitalized COVID-19 patients in the Montefiore Health System (Bronx, NY). We examined electrocardiograms (ECG) pre/post-medication initiation to evaluate QTc, HR, QRS duration, and presence of other arrhythmias.

Results One hundred five patients (mean age 67 years; $44.8 \% \mathrm{~F}$ ) were analyzed. The median time from the first dose of any treatment to post-medication ECG was 2 days (IQR: 1-3). QTc in men increased from baseline (440 vs $455 \mathrm{~ms}, p<0.001$ ), as well as in women (438 vs $463 \mathrm{~ms}, p<0.001$ ). The proportion of patients with QT prolongation increased significantly $(14.3 \%$ vs $34.3 \%, p<0.001)$ even when adjusted for electrolyte abnormalities. The number of patients whose QTc $\geq 500 \mathrm{~ms}$ was significantly increased after treatment ( $16.2 \%$ vs. $4.8 \%, p<0.01)$. Patients with either QTc $\geq 500 \mathrm{~ms}$ or an increase of $60 \mathrm{~ms}$ had a higher frequency of death (47.6\% vs. $22.6 \%, p=0.02$ ) with an odds ratio of 3.1 (95\% CI: 1.1-8.7). Adjusting for race/ethnicity yielded no significant associations.

Conclusions Hydroxychloroquine, chloroquine, and/or azithromycin were associated with QTc prolongation but did not result in fatal arrhythmias. Our findings suggest that any harm is unlikely to outweigh potential benefits of treatment. Careful risk-benefit analyses for individual patients should guide the use of these medications. Randomized control trials are necessary to evaluate their efficacies.
\end{abstract}

Keywords SARS-CoV-2 $\cdot$ COVID-19 $\cdot$ QT $\cdot$ Hydroxychloroquine $\cdot$ Chloroquine $\cdot$ Azithromycin

\author{
Andrew Krumerman \\ akrumerm@montefiore.org \\ Brian C Hsia \\ brian.c.hsia@gmail.com \\ Nicolas Greige \\ ngreige94@gmail.com \\ Jose A Quiroz \\ jose.quiroz@einsteinmed.org \\ Ahmed S Khokhar \\ ahmed.khokhar@einsteinmed.org \\ Johanna Daily \\ jdaily@montefiore.org \\ Luigi Di Biase \\ ldibiase@montefiore.org \\ Kevin J Ferrick \\ kferrick@montefiore.org
}

John D Fisher

jfisher@montefiore.org

1 Division of Cardiology, Department of Medicine, Albert Einstein College of Medicine \& Montefiore Medical Center, 111 East 210th Street, Room N2, Bronx, NY 10467, USA

2 Harold and Muriel Block Institute for Clinical and Translational Research, Albert Einstein College of Medicine \& Montefiore Medical Center, Bronx, NY, USA

3 Department of Medicine, Albert Einstein College of Medicine \& Montefiore Medical Center, Bronx, NY, USA

4 Division of Infectious Diseases, Department of Medicine, Albert Einstein College of Medicine \& Montefiore Medical Center, Bronx, NY, USA

5 Department of Microbiology \& Immunology, Albert Einstein College of Medicine \& Montefiore Medical Center, Bronx, NY, USA 


\section{Introduction}

The novel 2019 coronavirus, severe acute respiratory syndrome coronavirus 2 (SARS-CoV-2), commonly known as COVID-19, has become a global pandemic with nearly 2 million cases worldwide and over 100,000 deaths as of April 12, $2020[1,2]$. New York City specifically has become recognized as a global epicenter of the pandemic with the most deaths in the country and total case count over 100,000 [1]. Initial studies from Asia have revealed that mortality from COVID-19 increases with advanced age, comorbid conditions, presence of dyspnea, hypoxia, leukocytosis, lymphopenia, elevated CRP, high sequential organ failure assessment score, and elevated d-dimer [3-7]. These patients commonly experienced end organ damage such as acute respiratory distress syndrome, acute cardiac or kidney injury, shock, or disseminated intravascular coagulation prior to death $[3,4,7]$. The pandemic has disproportionately impacted minority populations with higher rates of poor outcomes [8,9]. Several drugs are being studied for COVID-19 including chloroquine, hydroxychloroquine, and azithromycin, resulting in national shortages [10-12].

The need for an effective COVID-19 treatment is becoming increasingly urgent. However, the rapid and extensive administration of these drugs will yield some risks and adverse outcomes as well, notably impacting cardiovascular status via arrhythmia induction. Hydroxychloroquine and chloroquine have both been associated with QT prolongation, thus increasing the risk of torsade de pointes, ventricular arrhythmias, and sudden cardiac death [13-16]. Azithromycin has also demonstrated QT prolongation among patients, with older age as a risk factor [17-20]. The higher incidence of QT prolongation among older patients is particularly concerning in the setting of COVID-19 treatment given that they are also particularly susceptible to severe infection $[5$, 21]. Given the lack of data of these medications in COVID19, various guidelines have been published to assist physicians in navigating the risk-benefit analysis of medication administration. These guidelines suggest cessation of QTprolonging agents at a QTc $\geq 500 \mathrm{~ms}$ indicating diminished repolarization reserve or an increase in the QTc by $60 \mathrm{~ms}$ indicating a potent QTc reactor $[22,23]$.

The compendium of literature for COVID-19 is slowly growing but remains sparse. In studying these medications in COVID-19, it will become imperative to note the potential harm. COVID-19 patients in Wuhan had high rates of cardiac injury, and cardiac injury was a risk factor for mortality [21, 24]. It is possible that these medications may exacerbate and worsen outcomes in a subset of patients. With our study, we hope to ascertain the rate of developing QT prolongation among patients diagnosed with COVID-19, receiving chloroquine, hydroxychloroquine, and/or azithromycin in a largely minority population. Our primary hypothesis is that COVID-
19 patients receiving these medications are more likely to experience QT prolongation. We aim to evaluate the proportion of patients that develop clinically significant QT prolongation and subsequent outcomes.

\section{Methods}

We conducted an observational study of adult subjects hospitalized in the Montefiore Health System and diagnosed with novel coronavirus, COVID-19. Subjects were adults 18 years or older who met the following criteria: (a) received a positive SARS-CoV-2 test result confirmed by RT-PCR; (b) hospitalized within the Montefiore Health System; (c) received chloroquine $(250 \mathrm{mg}$ or $500 \mathrm{mg}$ ), hydroxychloroquine $(400 \mathrm{mg}$ ), and/or azithromycin $(250 \mathrm{mg}$ or $500 \mathrm{mg}$ ) for treatment of COVID-19 infection; (d) had a standard 12-lead, 10 s electrocardiogram (ECG) before and after COVID-19 treatment initiation. Treatment dosing and schedule were as per standard hospital protocol, based on the available literature, for COVID-19: hydroxychloroquine $400 \mathrm{mg}$ twice on the 1st day, followed by once per day for 4 days afterward or as determined by the responsible physician; azithromycin, based on pneumonia treatment guidelines, $500 \mathrm{mg}$ on the 1st day, followed by $250 \mathrm{mg}$ once per day for 4 days afterward [25, 26]. We excluded patients that were under the age of 18 , had only a single ECG or did not have an ECG after medication initiation, or did not receive the medications of interest. Approval was obtained from the Institutional Review Board at the Albert Einstein College of Medicine, Bronx, New York.

Patient ECGs were examined using the MUSE database (General Electric Healthcare, Chicago, IL). The timestamp of ECGs was crosschecked with medication administration recorded in the patient's chart to ensure that baseline ECGs were obtained prior to medication initiation, and postmedication ECGs were obtained after medication initiation. The first ECG from the hospitalization served as the baseline ECG for each patient. If there was not an ECG prior to medication initiation for that hospitalization, the most recent ECG from a prior hospitalization was used as the baseline ECG.

The primary outcome of interest for this study was the QTc interval. The following information was obtained from each ECG: heart rate, QRS duration, QT interval, the presence of torsade de pointes (TdP), ventricular tachycardia/fibrillation, atrial flutter/fibrillation, supraventricular tachycardia, right bundle branch block (RBBB), and left bundle branch block (LBBB). Computer interpretation utilizing the 12SL algorithm was verified by a board-certified cardiologist [27]. When there was a discrepancy between the automated reading and the interpreter's measurement of the QT interval, adjudication was performed by a second interpreter. The corrected QT interval (QTc) was calculated using Bazett's formula. 
Patient charts were queried using Clinical Looking Glass (Montefiore Health System, Bronx, NY) to obtain demographic data, comorbidities, outpatient medication use, ejection fraction, body mass index, and electrolyte data. The closest electrolyte values within a 24 -h period of the baseline and post-medication ECGs were obtained. Hypokalemia, hypocalcemia, and hypomagnesemia were defined as the following per our lab's convention: $\mathrm{K}<3.5 \mathrm{mEq} / \mathrm{L}, \mathrm{Ca}<8.5 \mathrm{mEq} / \mathrm{L}$, and $\mathrm{Mg}<1.7 \mathrm{mEq} / \mathrm{L}$. Mortality data was obtained directly from the patient charts.

\subsection{Statistical analysis}

All analyses were computed using Stata Version 16.0 (College Station, TX: StataCorp LLC). Bivariate analyses of paired data were performed using the McNemar test, paired $t$ test, or Wilcoxon signed-rank test. Unpaired data were analyzed using the chi-square test, and homogeneity of stratumspecific odds ratios was evaluated using the Breslow-Day test. All test assumptions were assessed, and violations of test assumptions were appropriately addressed. Alpha was set at the 0.05 level.

\section{Results}

\subsection{Participant demographics and characteristics}

Five hundred eighteen charts of consecutive patients suspected of COVID-19 infection and underwent COVID19 diagnostic testing were reviewed. Four hundred thirteen patients were excluded from the final analysis due to the following: 340 had either no baseline or follow-up ECG; 54 had a negative SARS-CoV-2 result; 18 did not receive hydroxychloroquine, chloroquine, or azithromycin; and 1 did not complete SARS-CoV-2 diagnostic testing (Fig. 1).

A total of 105 patients that were hospitalized between February 13, 2020, and April 5, 2020, were analyzed and included in this study. The study sample consisted of $44.8 \%$ females and had an average age of $67 \pm 15$ years (Table 1). The majority of participants identified as Non-Hispanic Black (40.9\%), Hispanic (31.4\%), or White (11.4\%). The prevalence of medical comorbidities was substantial, as $48.6 \%$ of patients had a history of hypertension, $39.0 \%$ had a history of diabetes mellitus, $28.6 \%$ had a history of chronic kidney disease, and $19.0 \%$ had a history atrial fibrillation. Additionally, $4.8 \%$ of patients had a history of myocardial infarction and $5.7 \%$ had cardiomyopathy, with the mean ejection fraction being $58.7 \pm$ $10.5 \%$. Furthermore, the mean body mass index (BMI) for patients was $29.6 \pm 6.3 \mathrm{~kg} / \mathrm{m}^{2}$.

Treatment regimens varied between patients. The preponderance of patients was treated with hydroxychloroquine, either alone $(38.1 \%)$ or in combination with azithromycin

\section{8 consecutive admissions for suspected COVID-19 infection}

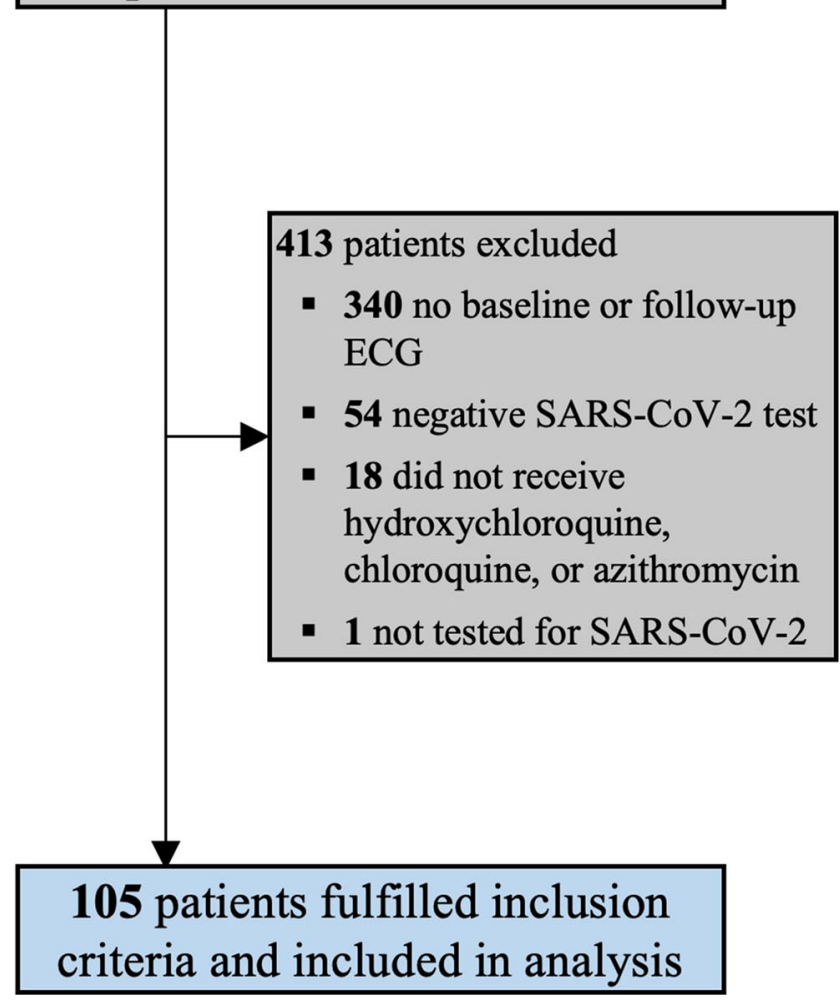

Fig. 1 Inclusion of COVID-19 patients in analysis

(31.4\%). The remainder of patients received azithromycin alone $(20.0 \%)$, chloroquine alone $(4.8 \%)$, or a combination of medications.

No patients received class I antiarrhythmics, and only $4.8 \%$ received class III antiarrhythmics between their pre- and postmedication ECGs. A minority of patients received other QTprolonging agents include ondansetron $(10 \%)$ and methadone $(3 \%)$. In this patient population, $32.4 \%$ were intubated, and $27.6 \%$ died due to respiratory complications from COVID-19. There were no cases of mortality due to fatal arrhythmias (i.e., $\mathrm{TdP}$, ventricular tachycardia/fibrillation).

\subsection{Electrocardiogram characteristics}

The median time from the first dose of chloroquine, hydroxychloroquine, or azithromycin to post-medication ECG was 2 (IQR: 1-3, range 0-18) days (Table 2). As compared to their baseline QTc, males had a significantly increased QTc after treatment administration (440 [IQR: $423-$ $457]$ vs. 455 [438-483] ms, $p<0.001$ ); females also experienced a similar significant increase in QTc as compared to baseline (438 [IQR: 423-460] vs. 463 [434-484] ms, $p<0.001)$. The median difference between baseline and post-medication QTc was 16 (IQR: -2-40) ms, with $12.4 \%$ of patients experiencing at least a $60 \mathrm{~ms}$ increase in QTc. 
Table 1 Patient characteristics

\begin{tabular}{|c|c|c|}
\hline Parameter ( $n=105$ patients) & Descriptive statistic & \\
\hline Age, year & $67 \pm 15$ & {$[29,97]$} \\
\hline \multicolumn{3}{|l|}{ Sex } \\
\hline Male & $58(55.2)$ & \\
\hline Female & $47(44.8)$ & \\
\hline Body mass index, $\mathrm{kg} / \mathrm{m} 2$ & $29.6 \pm 6.3$ & {$[17.1,48.7]$} \\
\hline \multicolumn{3}{|l|}{ Race/ethnicity } \\
\hline Hispanic & $33(31.4)$ & \\
\hline Non-Hispanic White & $12(11.4)$ & \\
\hline Non-Hispanic Black & $43(40.9)$ & \\
\hline Non-Hispanic Asian & $3(2.9)$ & \\
\hline Non-Hispanic Other & $6(5.7)$ & \\
\hline Unknown & $8(7.6)$ & \\
\hline \multicolumn{3}{|l|}{ Comorbidities } \\
\hline Hypertension & $51(48.6)$ & \\
\hline Diabetes mellitus & $41(39.0)$ & \\
\hline Cardiomyopathy & $6(5.7)$ & \\
\hline Prior MI & $5(4.8)$ & \\
\hline Chronic kidney disease & $30(28.6)$ & \\
\hline Prior atrial fibrillation & $20(19.0)$ & \\
\hline \multicolumn{3}{|l|}{ Other QT prolonging agents during hospitalization ${ }^{1}$} \\
\hline Class I antiarrhythmics & $0(0.0)$ & \\
\hline Class III antiarrhythmics ${ }^{2}$ & $5(4.8)$ & \\
\hline Ondansetron & $11(10.5)$ & \\
\hline Methadone & $3(2.9)$ & \\
\hline Ejection fraction, $\%$ (most recent if available) & $58.7 \pm 10.5$ & {$[30,80]$} \\
\hline \multicolumn{3}{|l|}{ Treatment } \\
\hline Chloroquine & $5(4.8)$ & \\
\hline Hydroxychloroquine & $40(38.1)$ & \\
\hline Azithromycin & $21(20.0)$ & \\
\hline Chloroquine and hydroxychloroquine & $1(1.0)$ & \\
\hline Chloroquine and azithromycin & $4(3.8)$ & \\
\hline Hydroxychloroquine and azithromycin & $33(31.4)$ & \\
\hline Chloroquine, hydroxychloroquine, and azithromycin & $1(1.0)$ & \\
\hline Intubated & $34(32.4)$ & \\
\hline Mortality & $29(27.6)$ & \\
\hline
\end{tabular}

Reported as mean $\pm \mathrm{SD}$ or $n(\%)$. Ranges reported for continuous variables

${ }^{1}$ Recorded if medication was administered between pre- and post-medication ECG

${ }^{2} 1$ patient on dofetilide and 4 patients on amiodarone

Additionally, the number of patients whose QTc $\geq 500 \mathrm{~ms}$ was significantly increased after treatment (16.2 vs. $4.8 \%$, $p<0.01)$. When QTc prolongation was defined as $\geq 470 \mathrm{~ms}$ for males and $\geq 480 \mathrm{~ms}$ for females, patients demonstrated a significantly greater frequency of QTc prolongation after medication administration as compared to baseline $(14.3 \%$ vs $34.3 \%, p<0.001)$ [22]. Of note, there was no significant association between ethnicity/race and either QTc prolongation $(p=0.12)$, change in QTc $\geq 60 \mathrm{~ms}(p=0.55)$ QTc $\geq$ $500 \mathrm{~ms}(p=0.63)$, or mortality $(p=0.48)$. Furthermore, stratification by the presence of either hypokalemia, hypocalcemia, or hypomagnesemia did not alter the statistical significance of the aforementioned increase in QT prolongation, and the Breslow-Day test of homogeneity was not significant ( $p=$ 0.39 ) which suggests that the association between treatment and QTc prolongation exists irrespective of electrolyte imbalance.

QTc pre- and post-treatment were also stratified by intubation status and mortality. Median baseline QTc was significantly greater in patients that were intubated vs those that were 
Table 2 ECG Characteristics

\begin{tabular}{|c|c|c|c|}
\hline$n=105$ & Baseline ECG & Post-medication ECG & $P$ value $^{1}$ \\
\hline $\mathrm{HR}>120$ & $9(8.6)$ & $11(10.5)$ & 0.77 \\
\hline Atrial flutter & $1(1.0)$ & $2(1.9)$ & $>0.99$ \\
\hline Atrial fibrillation & $8(7.6)$ & $10(9.5)$ & 0.69 \\
\hline Supraventricular tachycardia & $1(1.0)$ & $3(2.8)$ & 0.5 \\
\hline Right bundle branch block & $9(8.6)$ & $12(11.4)$ & 0.38 \\
\hline Left bundle branch block & $0(0.0)$ & $2(1.9)$ & 0.5 \\
\hline Non-specific intraventricular conduction block & $4(3.8)$ & $0(0.0)$ & 0.13 \\
\hline Torsade de pointes & $0(0.0)$ & $0(0.0)$ & - \\
\hline Ventricular tachycardia & $0(0.0)$ & $1(1.0)$ & $>0.99$ \\
\hline Ventricular fibrillation & $0(0.0)$ & $0(0.0)$ & - \\
\hline $\begin{array}{l}\text { \#Days after drug initiation (post-drug ECG only), } \\
\quad n \text { [min, } \max ]\end{array}$ & - & $\begin{array}{l}2(1-3) \\
{[0,18]}\end{array}$ & \\
\hline \multicolumn{4}{|l|}{ QTc, ms, median (IQR) } \\
\hline Male & $440(423-457)$ & $455(438-483)$ & $<0.001$ \\
\hline Female & $438(423-460)$ & $463(434-484)$ & $<0.001$ \\
\hline Difference between baseline and post-drug QTc, ms & - & $16(-2-40)$ & \\
\hline QTc prolongation $^{2}$ & $15(14.3)$ & $36(34.3)$ & $<0.001$ \\
\hline $\mathrm{QTc} \geq 500 \mathrm{~ms}$ & $5(4.8)$ & $17(16.2)$ & $<0.01$ \\
\hline Change in QTc $\geq 60 \mathrm{~ms}$ & - & $13(12.4)$ & \\
\hline Met criteria for medication discontinuation ${ }^{3}$ & - & $21(20.0)$ & \\
\hline Potassium $(n=87)^{4}$ & $4.4 \pm 0.7$ & $4.4 \pm 0.7$ & 0.61 \\
\hline Calcium $(n=95)^{4}$ & $8.7 \pm 0.6$ & $8.4 \pm 0.6$ & $<0.001$ \\
\hline Magnesium $(n=54)^{4}$ & $2.1(1.9-2.3)$ & $2.1(1.9-2.4)$ & 0.17 \\
\hline
\end{tabular}

Reported as mean $\pm \mathrm{SD}$, median (IQR), or $n(\%)$

${ }^{1}$ McNemar's test was used to analyze differences in HR > 120, atrial flutter/fibrillation, SVT, RBBB, LBBB, $\mathrm{TdP}$, ventricular tachycardia/fibrillation, proportion of people with QTc prolongation, and QTc $\geq 500 \mathrm{~ms}$; Wilcoxon signed-rank test was used to analyze QTc and magnesium levels; paired $t$ test was used to analyze potassium and calcium levels

${ }^{2}$ QT prolongation defined as QTc $\geq 470 \mathrm{~ms}$ in men and $\geq 480 \mathrm{~ms}$ in women

${ }^{3}$ Criteria for medication discontinuation was defined as having a post-drug ECG QTc $\geq 500 \mathrm{~ms}$ or an increase from baseline of $\geq 60 \mathrm{~ms}$

${ }^{4} \mathrm{~N}$ refers to total number of complete pairs that were analyzed

not intubated (449 vs. $433 \mathrm{~ms}, p<0.01$ ), but this association was not significant post-medication (466 vs. $454 \mathrm{~ms}, p=$ 0.13 ). Intubated patients, as compared to non-intubated patients, also had a significantly greater prevalence of QTc prolongation at baseline (10 [29.4\%] vs. 5 [7.0\%], $p<0.01)$, but this association was not significant post-medication administration $(14[41.2 \%]$ vs. 22 [31.0\%], $p=0.30)$. Both median baseline QTc (467 vs. $436 \mathrm{~ms}, p<0.01$ ) and post-treatment QTc (481 vs. $454 \mathrm{~ms}, p<0.01$ ) were significantly greater in deceased patients vs non-deceased patients. Deceased patients vs non-deceased patients also had a significantly greater prevalence of QTc prolongation both at baseline (10 [34.5\%] vs. 5 $[6.6 \%], p<0.001)$ and after $(16[55.2 \%]$ vs. 20 [26.3\%], $p<$ $0.01)$ treatment.

Current treatment guidelines recommend cessation of QTprolonging medications after observing either an increase of QTc $\geq 60 \mathrm{~ms}$ or a QTc $\geq 500 \mathrm{~ms}[22,23]$. Twenty percent of patients in our sample met these criteria for discontinuation at the time of post-treatment ECG. The frequency of mortality among those meeting medication discontinuation criteria was greater than those that did not meet criteria (47.6 vs. $22.6 \%$, $p=0.02$ ), with an unadjusted odds ratio of 3.1 (95\% CI: $1.1-$ 8.7). This can be interpreted to mean that patients with an increase in QTc $\geq 60 \mathrm{~ms}$ from baseline or a QTc $\geq 500 \mathrm{~ms}$ had a 3.1 times increased odds of mortality than those not exceeding the aforementioned parameters. Intubated patients did not have a significantly different prevalence of meeting treatment discontinuation criteria as compared to nonintubated patients (8 [23.5\%] vs. 13 [18.3\%], $p=0.53)$.

The prevalence of tachycardia, atrial flutter, atrial fibrillation, supraventricular tachycardia, right or left bundle branch block, and nonspecific intraventricular conduction block did not significantly differ between baseline and post-treatment ECG. Additionally, there was no evidence of torsade de 
pointes or ventricular fibrillation in this sample. A single patient was noted to have stable ventricular tachycardia on posttreatment ECG.

\section{Discussion}

The COVID-19 pandemic has resulted in a substantial study of chloroquine, hydroxychloroquine, and azithromycin. The anti-viral activity and immunomodulating effects of chloroquine and hydroxychloroquine are potentially valuable in dampening pro-inflammatory cytokines, given evidence of cytokine storm in fatal cases [10, 25, 28-31]. Azithromycin and hydroxychloroquine combination resulted in $100 \%$ clearance of viral load in respiratory secretions of 36 patients, and reports from China suggest chloroquine may result in pneumonia inhibition and truncated disease courses [32, 33]. However, a recent observational study found no association between hydroxychloroquine and rates of intubation or mortality [34].

Continued research delineating the effects of these medications remains necessary in the COVID-19 pandemic. A preliminary, non-peer-reviewed analysis at the New York University found a high prevalence of QTc $>500 \mathrm{~ms}$ at $11 \%$ in a sample of 84 patients, with renal failure being a strong risk factor, although they did not demonstrate an association with mortality [35]. A few other observational studies of small sample sizes have also established an association with QT prolongation and these medications [36-38]. No studies, however, have been performed in vulnerable minority populations. Refining treatment strategies in these populations remains a priority given reports of worse outcomes [8, 9]. In this study, we evaluate the impact of hydroxychloroquine, chloroquine, and azithromycin on QT prolongation and the impact on mortality with high-risk QT prolongation in a multiethnic/racial population.

This sample of patients was representative of the larger Bronx population typically seen in the Montefiore Health System with minority populations comprising a significant proportion [39]. A large proportion of our population was older and suffered from several comorbidities with hypertension and diabetes being the most common followed by CKD, similar to previously described demographics of at-risk COVID-19 patients [4, 5, 21]. Additionally, obese patients comprised a significant portion of our patient sample conferring additional risk for poor outcomes, with a recent study identifying BMI $>30 \mathrm{~kg} / \mathrm{m}^{2}$ as a risk factor for hospitalization and ICU admission among patients under 60 years old [40].

Among our patients, we found the median QTc in both men and women to be significantly increased. Additionally, the proportion of men and women that met the criteria of QTc prolongation using the cutoffs of $\geq 470 \mathrm{~ms}$ and $\geq 480 \mathrm{~ms}$ increased significantly relative to baseline $(14.3 \%$ vs $34.3 \%, p<0.001)$, even when controlling for electrolyte abnormalities. The proportion of patients on antiarrhythmics known to prolong the QT interval, class I, and III medications, was low (4.8\%) thus conferring minimal impact on our results. The overall incidences of RBBB, LBBB, and non-specific intraventricular conduction block were also low and not significantly different between pre- and post-medication ECGs, making these arrhythmias unlikely contributors to the impact on QTc prolongation. Given this data, it is reasonable to conclude that the administration of hydroxychloroquine, chloroquine, and/or azithromycin likely confer a risk for QTc prolongation. However, there were no cases of TdP or ventricular fibrillation, and only one patient with ventricular tachycardia. Additionally, all fatal COVID19 cases in this sample were due to respiratory complications. Thus, while there may be a statistically significant increased risk of developing prolonged QTc in patients treated with these medications, the clinical risk of harm or death may be outweighed by the potential benefit of treating the infection for the vast majority of patients. To date, there are no randomized controlled trials demonstrating efficacy data for hydroxychloroquine. Additional studies are necessary to determine the exact risk-benefit ratio for such therapy.

In the absence of concrete data for management of COVID-19 patients, recent expert guidelines have proposed using cutoffs of QTc $\geq 500 \mathrm{~ms}$ or an increase of QTc $\geq 60 \mathrm{~ms}$ to discontinue these agents, identifying patients with possible diminished repolarization reserve or a repolarization reactor, and thus at high risk for fatal ventricular arrhythmias [22, 23]. Of note, a minority of patients had QTc $>500 \mathrm{~ms}$ at baseline $(4.8 \%)$. This minority of patients tended to have widened QRS complexes due to the baseline bundle branch block at baseline. Additionally, treatment administration in these patients was at the discretion of the primary team, and no patients in the entire cohort experienced fatal arrhythmias. We identified 21 patients (20\%) in our sample that fulfilled criteria for medication discontinuation, and the frequency of mortality was nearly double those who did not meet these criteria (47.6\% vs $22.6 \%, p=0.02)$, with an OR of 3.1 (95\% CI: 1.1-8.7). Notably, the deceased group also had higher baseline and post-treatment QTc relative to the rest of the cohort. There are, however, several other factors contributing to the higher odds of mortality in this group of patients. This population suffered from a greater burden of infection with severe disease, supported by the high intubation rate in the entire cohort $(32.4 \%)$. It is likely that during the course of hospitalization, significant clinical events occurred that resulted in QT prolongation and increased mortality such as cardiac ischemia or myocarditis. However, troponins for this cohort were not routinely collected, thus precluding an analysis of myocardial damage. Although no patients died from fatal arrhythmias, these findings support that QTc may actually be considered a vital sign and marker for all-cause mortality, rather than just a risk factor for arrhythmias [41]. 
Of the approximately 500 consecutive charts reviewed, only $20 \%$ of individuals received baseline and follow-up ECGs to ensure QT interval stability. Many patients not included in this analysis had QT interval monitoring via continuous telemetry or patch telemetry recordings. Limited staff and personal protective equipment likely resulted in 12-lead ECG monitoring of the sickest patients. Our data highlights the importance of standardized pre- and post-treatment ECG monitoring in COVID-19 patients. This is especially important when patients are treated with multiple QT-prolonging agents, such as ondansetron and methadone (10\% and $3 \%$ respectively in our study).

The CDC's monitoring program COVID-NET suggested that black patients are disproportionately impacted by COVID-19 [9]. In our study, we found no statistically significant associations between ethnicity/race and QTc prolongation, QTc $>500 \mathrm{~ms}$, or mortality. However, given our study's sample size, a true association cannot be excluded. A larger study will be necessary to ascertain the risk of mortality among racial minorities.

The main limitation of this study was selection bias. The population included in this study includes only patients that required hospitalization. Moreover, patients monitored with serial 12-lead ECG recording were more likely to be in an ICU or specialized unit where more resources for serial testing were available. This is supported by the high intubation rate in our cohort and may contribute to the observed mortality rate. Additionally, intubation and mortality rates likely reflect the selection of the sicker COVID-19 infected population. Despite this, treatment with hydroxychloroquine did not appear to cause harmful proarrhythmic events. Additional prospective controlled studies will be necessary to truly determine the risk-benefit ratio of such therapy. Arrhythmia monitoring should be more routinely implemented and incorporated into institutional protocols for more patients receiving these medications as is suggested by other guidelines [22, 23]. Enacting such policies will allow further, larger studies to support the conclusions made by this study.

\section{Conclusions}

Administration of hydroxychloroquine, chloroquine, and/or azithromycin for treatment of COVID-19 resulted in statistically significant increases in QTc and increased the proportion of patients with QTc prolongation even after controlling for electrolyte abnormalities. QT interval prolongation appeared to be consistent regardless of ethnicity or race. Patients who had either QTc $\geq 500 \mathrm{~ms}$ or an increase in QTc of $\geq 60 \mathrm{~ms} \mathrm{had}$ increased odds of mortality than those who did not, though no patients died from ventricular tachyarrhythmia. Based on these findings, patients with QTc prolongation at baseline may be at increased risk for arrhythmias and should be monitored more closely. Risk-benefit analyses should be carefully considered for each patient. RCTs are necessary to further inform the use of these medications in COVID-19 patients.

Availability of data and materials Not applicable.

Author contributions All authors contributed to the study conception and design. Material preparation and data collection were performed by Brian Hsia, Jose Quiroz, and Ahmed Khokhar. Analysis was performed by Brian Hsia and Nicolas Greige. The first draft of the manuscript was written by Brian Hsia and Nicolas Greige. All authors commented on previous versions of the manuscript. All authors read and approved the final manuscript.

\section{Compliance with ethical standards}

Conflict of interest No conflicts to disclose.

Ethical approval Obtained from the Institutional Review Board at the Albert Einstein College of Medicine, Bronx, New York.

Consent to participate Not applicable.

Consent for publication Not applicable.

Code availability Not applicable.

\section{References}

1. Center for Systems Science and Engineering at Johns Hopkins University. Coronavirus COVID-19 Dashboard by the Center for Systems Science and Engineering at Johns Hopkins University. https://coronavirus.jhu.edu/map.html.

2. Dong E, Du H, Gardner L. An interactive web-based dashboard to track COVID-19 in real time. Lancet Infect Dis. 2020;20:533-4. https://doi.org/10.1016/S1473-3099(20)30120-1.

3. Deng Y, Liu W, Liu K, Fang YY, Shang J, Zhou L, et al. Clinical characteristics of fatal and recovered cases of coronavirus disease 2019 (COVID-19) in Wuhan, China: a retrospective study. Chin Med J. 2020;133:1261-7. https://doi.org/10.1097/CM9. 0000000000000824.

4. Goh KJ, Choong MC, Cheong EH, Kalimuddin S, Duu Wen S, Phua GC, et al. Rapid progression to acute respiratory distress syndrome: review of current understanding of critical illness from COVID-19 infection. Ann Acad Med Singap. 2020;49(1):1-9.

5. Mo P, Xing Y, Xiao Y, Deng L, Zhao Q, Wang H, et al. Clinical characteristics of refractory COVID-19 pneumonia in Wuhan. China Clin Infect Dis. 2020. https://doi.org/10.1093/cid/ciaa270.

6. Zhou F, Yu T, Du R, Fan G, Liu Y, Liu Z, et al. Clinical course and risk factors for mortality of adult inpatients with COVID-19 in Wuhan, China: a retrospective cohort study. Lancet. 2020;395: 1054-62. https://doi.org/10.1016/S0140-6736(20)30566-3.

7. Wu C, Chen X, Cai Y, Xia J, Zhou X, Xu S, et al. Risk factors associated with acute respiratory distress syndrome and death in patients with coronavirus disease 2019 pneumonia in Wuhan. China JAMA Intern Med. 2020. https://doi.org/10.1001/ jamainternmed.2020.0994.

8. Haynes N, Cooper LA, Albert MA, Association of Black C. At the heart of the matter: unmasking and addressing COVID-19's toll on 
diverse populations. Circulation. 2020. doi:https://doi.org/10.1161/ CIRCULATIONAHA.120.048126.

9. Garg S, Kim L, Whitaker M, O'Halloran A, Cummings C, Holstein $\mathrm{R}$, et al. Hospitalization rates and characteristics of patients hospitalized with laboratory-confirmed coronavirus disease 2019 COVID-NET, 14 states, march 1-30. In: MMWR Morb Mortal Wkly Rep. Centers for Disease Control and Prevention; 2020. https://www.cdc.gov/mmwr/volumes/69/wr/mm6915e3.htm?s_ cid $=$ mm6915e $3 \mathrm{w}$.

10. Zhou D, Dai SM, Tong Q. COVID-19: a recommendation to examine the effect of hydroxychloroquine in preventing infection and progression. J Antimicrob Chemother. 2020;75:1667-70. https:// doi.org/10.1093/jac/dkaa114.

11. US Food \& Drug Administration. FDA Drug Shortages. https:// www.accessdata.fda.gov/scripts/drugshortages/default.cfm.

12. Vaduganathan M, van Meijgaard J, Mehra MR, Joseph J, O'Donnell CJ, Warraich HJ. Prescription fill patterns for commonly used drugs during the COVID-19 pandemic in the United States. JAMA. 2020;323:2524. https://doi.org/10.1001/jama.2020.9184.

13. Chen CY, Wang FL, Lin CC. Chronic hydroxychloroquine use associated with QT prolongation and refractory ventricular arrhythmia. Clin Toxicol (Phila). 2006;44(2):173-5. https://doi.org/10. 1080/15563650500514558.

14. Khobragade SB, Gupta P, Gurav P, Chaudhari G, Gatne MM, Shingatgeri VM. Assessment of proarrhythmic activity of chloroquine in in vivo and ex vivo rabbit models. J Pharmacol Pharmacother. 2013;4(2):116-24. https://doi.org/10.4103/0976$500 X .110892$.

15. Stas P, Faes D, Noyens P. Conduction disorder and QT prolongation secondary to long-term treatment with chloroquine. Int $\mathrm{J}$ Cardiol. 2008;127(2):e80-2. https://doi.org/10.1016/j.ijcard.2007. 04.055 .

16. Bustos MD, Gay F, Diquet B, Thomare P, Warot D. The pharmacokinetics and electrocardiographic effects of chloroquine in healthy subjects. Trop Med Parasitol. 1994;45(2):83-6.

17. Choi Y, Lim HS, Chung D, Choi JG, Yoon D. Risk evaluation of azithromycin-induced QT prolongation in real-world practice. Biomed Res Int. 2018;2018:1574806-8. https://doi.org/10.1155/ 2018/1574806.

18. Zhang M, Xie M, Li S, Gao Y, Xue S, Huang H, et al. Electrophysiologic studies on the risks and potential mechanism underlying the proarrhythmic nature of azithromycin. Cardiovasc Toxicol. 2017;17(4):434-40. https://doi.org/10.1007/s12012-0179401-7.

19. Maisch NM, Kochupurackal JG, Sin J. Azithromycin and the risk of cardiovascular complications. J Pharm Pract. 2014;27(5):496500. https://doi.org/10.1177/0897190013516503.

20. Howard PA. Azithromycin-induced proarrhythmia and cardiovascular death. Ann Pharmacother. 2013;47(11):1547-51. https://doi. org/10.1177/1060028013504905.

21. Shi S, Qin M, Shen B, Cai Y, Liu T, Yang F, et al. Association of cardiac injury with mortality in hospitalized patients with COVID19 in Wuhan. China JAMA Cardiol. 2020. https://doi.org/10.1001/ jamacardio.2020.0950.

22. Guidicessi JR, Noseworthy PA, Friedman PA, Ackerman MJ. Urgent guidance for navigating and circumventing the QTc prolonging and torsadogenic potential of possible pharmacotherapies for COVID-19 [published online ahead of print march 25, 2020]. Mayo Clin Proc doi:https://doi.org/10.1016/j.mayocp. 2020.03.024, 2020.

23. Roden DM, Harrington RA, Poppas A, Russo AM. Considerations for drug interactions on QTc in exploratory COVID-19 (coronavirus disease 2019) treatment. Circulation. 2020;141:e906-7. https:// doi.org/10.1161/CIRCULATIONAHA.120.047521.

24. Guo T, Fan Y, Chen M, Wu X, Zhang L, He T, et al. Cardiovascular implications of fatal outcomes of patients with coronavirus disease
2019 (COVID-19). JAMA Cardiol. 2020. https://doi.org/10.1001/ jamacardio.2020.1017.

25. Yao X, Ye F, Zhang M, Cui C, Huang B, Niu $\mathrm{P}$, et al. In vitro antiviral activity and projection of optimized dosing design of hydroxychloroquine for the treatment of severe acute respiratory syndrome coronavirus 2 (SARS-CoV-2). Clin Infect Dis. 2020. https://doi.org/10.1093/cid/ciaa237.

26. Metlay JP, Waterer GW, Long AC, Anzueto A, Brozek J, Crothers $\mathrm{K}$, et al. Diagnosis and treatment of adults with communityacquired pneumonia. An official clinical practice guideline of the American Thoracic Society and Infectious Diseases Society of America. Am J Respir Crit Care Med. 2019;200(7):e45-67. https://doi.org/10.1164/rccm.201908-1581ST.

27. General Electric Healthcare. Marquette ${ }^{\mathrm{TM}}$ 12SL ${ }^{\mathrm{TM}}$ ECG Analysis Program Physician's Guide 2036070-006 Revision C, 2010. In: Company GE, editor.2012.

28. Devaux CA, Rolain JM, Colson P, Raoult D. New insights on the antiviral effects of chloroquine against coronavirus: what to expect for COVID-19? Int J Antimicrob Agents. 2020;105938:105938. https://doi.org/10.1016/j.ijantimicag.2020.105938.

29. Qin C, Zhou L, Hu Z, Zhang S, Yang S, Tao Y, et al. Dysregulation of immune response in patients with COVID-19 in Wuhan. China. Clin Infect Dis. 2020. https://doi.org/10.1093/cid/ciaa248.

30. Zhou P, Yang XL, Wang XG, Hu B, Zhang L, Zhang W, et al. A pneumonia outbreak associated with a new coronavirus of probable bat origin. Nature. 2020;579(7798):270-3. https://doi.org/10.1038/ s41586-020-2012-7.

31. Wang M, Cao R, Zhang L, Yang X, Liu J, Xu M, et al. Remdesivir and chloroquine effectively inhibit the recently emerged novel coronavirus (2019-nCoV) in vitro. Cell Res. 2020;30(3):269-71. https://doi.org/10.1038/s41422-020-0282-0.

32. Gautret P, Lagier JC, Parola P, Hoang VT, Meddeb L, Mailhe M, et al. Hydroxychloroquine and azithromycin as a treatment of COVID-19: results of an open-label non-randomized clinical trial. Int J Antimicrob Agents. 2020;105949:105949. https://oi.org/10. 1016/j.jjantimicag.2020.105949.

33. Gao J, Tian Z, Yang X. Breakthrough: chloroquine phosphate has shown apparent efficacy in treatment of COVID-19 associated pneumonia in clinical studies. Biosci Trends. 2020;14(1):72-3. https://doi.org/10.5582/bst.2020.01047.

34. Geleris J, Sun Y, Platt J, Zucker J, Baldwin M, Hripcsak G, et al. Observational study of hydroxychloroquine in hospitalized patients with Covid-19. N Engl J Med. 2020;382:2411-8. https://doi.org/ 10.1056/NEJMoa2012410.

35. Chorin E, Dai M, Shulman E, Wadhwani L, Bar Cohen R, Barbhaiya $\mathrm{C}$ et al. The QT interval in patients with SARS-CoV-2 infection treated with hydroxychloroquine/azithromycin. medRxiv. 2020:2020.04.02.20047050. doi:https://doi.org/10.1101/2020.04. 02.20047050

36. Saleh M, Gabriels J, Chang D, Kim BS, Mansoor A, Mahmood E, et al. The effect of chloroquine, hydroxychloroquine and azithromycin on the corrected QT interval in patients with SARSCoV-2 infection. Circ Arrhythm Electrophysiol. 2020;13:e08662. https://doi.org/10.1161/CIRCEP.120.008662.

37. Chorin E, Dai M, Shulman E, Wadhwani L, Bar-Cohen R, Barbhaiya C, et al. The QT interval in patients with COVID-19 treated with hydroxychloroquine and azithromycin. Nat Med. 2020;26:808-9. https://doi.org/10.1038/s41591-020-0888-2.

38. Mercuro NJ, Yen CF, Shim DJ, Maher TR, McCoy CM, Zimetbaum PJ, et al. Risk of QT interval prolongation associated with use of hydroxychloroquine with or without concomitant azithromycin among hospitalized patients testing positive for coronavirus disease 2019 (COVID-19). JAMA Cardiol. 2020. https:// doi.org/10.1001/jamacardio.2020.1834.

39. United States Census Bureau. United States Census Bureau: QuickFacts Bronx County (Bronx Borough), New York. 2019. 
https://www.census.gov/quickfacts/fact/table/ bronxcountybronxboroughnewyork/PST045217.

40. Lighter J, Phillips M, Hochman S, Sterling S, Johnson D, Francois

$\mathrm{F}$, et al. Obesity in patients younger than 60 years is a risk factor for

Covid-19 hospital admission. Clin Infect Dis. 2020. https://doi.org/ 10.1093/cid/ciaa415.

41. Haugaa KH, Bos JM, Tarrell RF, Morlan BW, Caraballo PJ, Ackerman MJ. Institution-wide QT alert system identifies patients with a high risk of mortality. Mayo Clin Proc. 2013;88(4):315-25. https://doi.org/10.1016/j.mayocp.2013.01.013.

Publisher's note Springer Nature remains neutral with regard to jurisdictional claims in published maps and institutional affiliations. 